\title{
Vibrations of beams with a variable cross-section fixed on rotational rigid disks
}

\section{Abstract}

The work is focused on the problem of vibrating beams with a variable cross-section fixed on a rotational rigid disk. The beam is loaded by a transversal time varying force orthogonal to an axis of the beam and simultaneously parallel to the disk's plane. There are many ways of usage of the technical moveable systems composed of elements with the variable cross-sections. The main applications are used in numerous types of turbines and pumps. The paper is a kind of introduction to the dynamic analysis of above mentioned beam systems. The equations of motion of rotational beams fixed on the rigid disks were derived. After introducing the Coriolis forces and the centrifugal forces, the transportation effect in the mathematical model was considered. This particular project is the first stage research, where there were proposed certain solutions of problems connected with the linear variable cross-sections systems. The further investigation considering the nonlinear systems has been proceeding. The results, analysis and comparison will be presented in the future works.

\section{Keywords}

variable cross-section, beam, rotary motion, dynamic flexibility

\section{Slawomir Zolkiewski*}

Silesian University of Technology, Institute of Engineering Processes Automation and Integrated Manufacturing Systems, Division of Mechatronics and Designing of Technical Systems, Faculty of Mechanical Engineering, 18a Konarskiego Street, 44-100 Gliwice, Poland

*Author email: slawomir.zolkiewski@polsl.pl

\section{INTRODUCTION}

The fundamental elements of the numerous mechanisms or machines, namely the beams and rods, are most commonly considered as the vibrating stationary systems. In these cases they are treated as the immovable ones without the coupling between the local oscillations and the main operational motion. The mechanical systems are predominantly considered both in the kinematical and dynamical aspects. The main problems resulting from the analysis are connected with the issues of controlling and stabilizing mechanical systems as such. Numerous research can be found in the literature $[1,3,4,7,9,14-16,18-22]$ and so far the solutions have been found by considering the transportation (considered as the main working motion) and the local oscillations separately $[2,3,5,6,8,10$ 12, 17]. Mesut Simsek in his paper [14] presents the nonlinear dynamic analysis of a functionally graded beam with pinned-pinned supports. Simsek uses the Timoshenko beam theory with the von Karman's nonlinear strain-displacement relationships. The beam was analyzed in relation to its 
variable thickness of a cross-section according to the power-law formula. The system of equations of motion is derived by using the Lagrange's equations. Transverse and axial deflections and rotation of the cross-sections of the beam were expressed in polynomial forms. The boundary conditions are taken into account by using the Lagrange multipliers. The Newmark-b method in conjunction with the direct iteration method was used to solve nonlinear equations of motion. In the work, large deflection, velocity of the moving load and excitation frequency on the beam displacements were analyzed. Some detailed cases were compared with each other. Another method of analysis of highspeed rotating beams is presented in [9] by Gunda. The author introduces a new finite element for free vibration analysis of rotating beams. The basis shape functions which apply a linear combination of the solution of the governing static differential equation of a stiff-string and a cubic polynomial are used. The introduced functions depend on the angular velocity and position of the analyzed section, what accounts for the centrifugal stiffening effect. In paper [1] the effects of rotary inertia were presented. The author shows the rotation impact on the extensional tensile force and the eigenvalues of beams. The analyzed beam was rotated uniformly about a transverse axis taking into account longitudinal elasticity. The perturbation technique and Galerkin's method were used. In the example of a typical helicopter rotor blade it was indicated that the extensional tensile force increases up to ten percent, when the rotary inertia contribution is retained in the modelling. Szefer in $[15,16]$ claimed that the vibrations from flexibility of elements of the mechanical composition are much smaller than a main dislocation of this composition. There is an increased range of velocities and accelerations of such a type systems. All those are caused by using more efficient drives or/and less and less weighting materials. In order to constraint power output of drives for motion of mechanical systems, materials with the lower mass density and stiffness are used. All those things are reasons for creating the new models of designing technical beamlike systems. The variable crosssection beams are applied in many practical applications nowadays. Both the linearly and nonlinearly variable cross-sections are used in technical systems. These systems should be taken under consideration with respect to a realistic distribution of mass and flexibility. Any variations in crosssections cannot be neglected and should be considered in the mathematical model assumptions. In spite of many works [e.g. 1, 9-12, 17, 22], where their authors analyzes the beams in rotations and beams with variable cross-sections, this work is devoted to general analyses both the linear and nonlinear ones of vibrating transversally beams with the variable cross-section. It is believed that new equations and results are presented in this paper.

\section{MODEL OF THE ANALYZED BEAMS FIXED ON THE ROTATIONAL RIGID DISK}

In this section the way of modelling of beams in rotational transportation is presented. The beam was assumed as the uniform one with the variable cross-section. The beam was fixed on the rotational disk that was assumed as a rigid element (Fig. 1). The disk rotates round its axis and the motion was described in two reference frames, the global stationary reference frame and the local one. The vibrations occurring in the local reference frame are transferred into the global reference frame with taking into consideration so called the transportation effect $[3,16,19,20]$. 


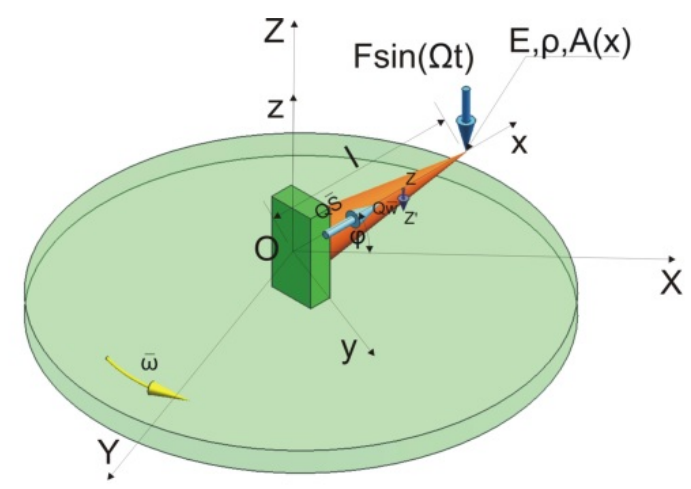

Fig. 1 Model of the analyzed beam on the rotational disk [3]

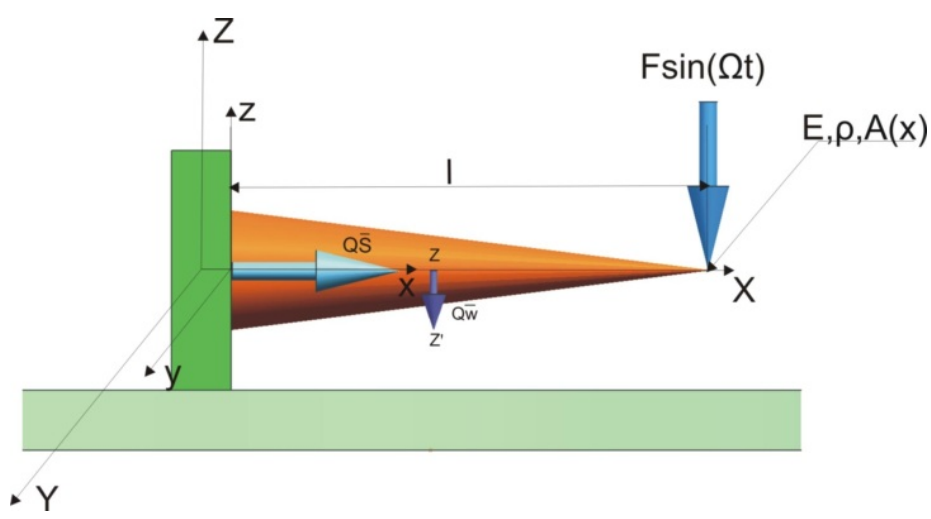

Fig. 2 Model of the analyzed beam on the rotational disk - the XZ projection [3]

where: $\rho$ - mass-density, $\mathrm{A}(\mathrm{x})$ - cross-section, $\mathrm{l}$ - length of the beam, $\mathrm{x}$ - location of the analyzed cross-section, $\boldsymbol{\omega}$ - angular velocity, $\Omega$ - frequency, $\mathbf{Q}$ - rotation matrix, $\mathbf{S}$ - position vector, $\mathbf{F}$ - harmonic force, E - Young modulus, w - vector of displacement.

There is defined a set of generalized coordinates allowing to make an assumption that generalized coordinates are the orthogonal projections on the coordinate axes of the global reference frame and can be written thus:

$$
\begin{aligned}
& q_{1}=r_{X}, \\
& q_{2}=r_{Y},
\end{aligned}
$$

and after differentiating (Eq. 1) and (Eq. 2) can be obtained the generalized velocities in the form:

$$
\begin{aligned}
& \dot{q}_{1}=\frac{d q_{1}}{d t}=\dot{r}_{X}=v_{X}, \\
& \dot{q}_{2}=\frac{d q_{2}}{d t}=\dot{r}_{Y}=v_{Y},
\end{aligned}
$$


or in the equivalent vector form expressing the displacements:

$$
\overline{\mathbf{r}}=\left[r_{X}, r_{Y}\right]^{T}
$$

and the equivalent vector expressing the velocities:

$$
\dot{\overline{\mathbf{r}}}=\left[\dot{r}_{X}, \dot{r}_{Y}\right]^{T} \text {. }
$$

The cross-section is dependent on the $x$ coordinate and for instance making an assumption that the analyzed beam is a cone (a base with radius equal $\mathrm{x} / \mathrm{l}$ ) it can be written as follows:

$$
A(x)=A_{0}\left(1-\frac{x}{l}\right)^{2}=\pi\left(1-\frac{x}{l}\right)^{2},
$$

The angular velocity of the rotational disk treated as the transportation velocity is defined as:

$$
\bar{\omega}=\left[\begin{array}{lll}
0 & 0 & \omega
\end{array}\right]^{T},
$$

The location of the analyzed cross-section is described by the position vector defined as follows:

$$
\overline{\mathbf{S}}=\left[\begin{array}{lll}
s & 0 & 0
\end{array}\right]^{T} .
$$

The xyz reference frame is the rotational one and the matrix allowing expressing vectors is the rotation matrix. The xyz local reference frame is fixed to the rotating beam and its z-axis coincides with the rotation axis of the rigid disk, thus the rotation matrix is defined as such:

$$
\mathbf{Q}=\left[\begin{array}{ccc}
\cos \varphi & -\sin \varphi & 0 \\
\sin \varphi & \cos \varphi & 0 \\
0 & 0 & 1
\end{array}\right]
$$

Different applications of such a type beam systems may have different boundary conditions and the cross-section can be considered as the linearly or nonlinearly variable one. For example the beam can be considered as the fixed at one end, the clamped-clamped one or with other boundary conditions etc. For example beams with one end clamped and the second free-free are considered (Fig. 1). The beam is fixed on the rotational rigid disk. An analyzed section of the beam is loaded by a harmonic time varying distributed force with unitary amplitude on a direction perpendicular to the centre line of the beam. The forces on origins of the beams are assumed as equal zero and also the displacements are equal zero, because the system is clamped in one end. Because of all these the specific boundary conditions for this beam should be written as follows: 


$$
\left\{\begin{array}{l}
w(0, t)=0 \\
\frac{\partial w(0, t)}{\partial x}=0 \\
\frac{\partial^{2} w(l, t)}{\partial x^{2}}=0 \\
\frac{\partial}{\partial x}\left[E I(x) \frac{\partial w^{2}(l, t)}{\partial x^{2}}\right]=-2 \int_{0}^{l} F_{0} \delta(x-l) \cos (\Omega t) d x
\end{array}\right.
$$

in every time moment $\mathrm{t} \geq 0$.

For the beams with the linearly variable cross-section the geometric moment of inertia can be assumed as the function proportional to the constant initial moment $I_{0}$ for example as:

$$
I(x)=I_{0}\left(1-\frac{x}{l}\right)^{4}
$$

After solving the boundary value problem, the eigenfunction for the displacement can be derived in the form:

$$
X(x)=x^{-\frac{n}{2}}\left[C_{1} J_{n}(k \sqrt{x})+C_{2} Y_{n}(k \sqrt{x})+C_{3} I_{n}(k \sqrt{x})+C_{4} K_{n}(k \sqrt{x})\right],
$$

where [13]:

$J_{n}$ - the Bessel function of the first kind, $Y_{n}$ - the Bessel function of the second kind, $I_{n}$ - the hyperbolic Bessel function of the first kind, $K_{n}$ - the hyperbolic Bessel function of the second kind, $C_{1,2,3,4}$ - the integrations constants, where: $n$ is a mode of vibrations of the beam with a variable cross-section. The $k$ coefficient is a linear approximation of the realistic eigenvalues of the analyzed systems.

\section{EQUATIONS OF MOTION OF THE ANALYZED SYSTEMS}

The equations of motion of the analyzed beamlike systems are obtained with the help of the classical method. The derived equations of motion can be presented in the matrix form as follows: 


$$
\begin{aligned}
& \rho A(x)\left[\begin{array}{ccc}
\cos \varphi & -\sin \varphi & 0 \\
\sin \varphi & \cos \varphi & 0 \\
0 & 0 & 1
\end{array}\right]\left[\begin{array}{c}
0 \\
0 \\
\frac{\partial^{2} w}{\partial t^{2}}
\end{array}\right]-\rho A(x)\left[\begin{array}{ccc}
\cos \varphi & -\sin \varphi & 0 \\
\sin \varphi & \cos \varphi & 0 \\
0 & 0 & 1
\end{array}\right]\left[\begin{array}{c}
\omega^{2} s \\
0 \\
0
\end{array}\right]+ \\
& +\rho A(x)\left[\begin{array}{ccc}
\cos \varphi & -\sin \varphi & 0 \\
\sin \varphi & \cos \varphi & 0 \\
0 & 0 & 1
\end{array}\right]\left[\begin{array}{c}
0 \\
\dot{\omega} s \\
0
\end{array}\right]=-E I(x)\left[\begin{array}{ccc}
\cos \varphi & -\sin \varphi & 0 \\
\sin \varphi & \cos \varphi & 0 \\
0 & 0 & 1
\end{array}\right]\left[\begin{array}{c}
0 \\
0 \\
\frac{\partial^{4} w}{\partial x^{4}}
\end{array}\right]+ \\
& -2 \frac{\partial E I(x)}{\partial x}\left[\begin{array}{ccc}
\cos \varphi & -\sin \varphi & 0 \\
\sin \varphi & \cos \varphi & 0 \\
0 & 0 & 1
\end{array}\right]\left[\begin{array}{c}
0 \\
0 \\
\frac{\partial^{3} w}{\partial x^{3}}
\end{array}\right]-\frac{\partial^{2} E I(x)}{\partial x^{2}}\left[\begin{array}{ccc}
\cos \varphi & -\sin \varphi & 0 \\
\sin \varphi & \cos \varphi & 0 \\
0 & 0 & 1
\end{array}\right]\left[\begin{array}{c}
0 \\
0 \\
\frac{\partial^{2} w}{\partial x^{2}}
\end{array}\right]
\end{aligned}
$$

in each point of the range $D=\{(x, t), x \in(0, l), t>0\}$ the Eq. (3.1) coincides with boundary conditions and initials conditions.

The projections of the equations of motion in the global reference frame are obtained from the Eq. (14) and in the XYZ global reference frame equations for individual axes can be expressed as:

$$
\begin{gathered}
\omega^{2} s \cos \varphi+\dot{\omega} s \sin \varphi=0 \\
\omega^{2} s \sin \varphi-\dot{\omega} s \cos \varphi=0 \\
\rho A(x) \frac{\partial^{2} w}{\partial t^{2}}+E I(x) \frac{\partial^{4} w}{\partial x^{4}}+2 \frac{\partial E I(x)}{\partial x} \frac{\partial^{3} w}{\partial x^{3}}+\frac{\partial^{2} E I(x)}{\partial x^{2}} \frac{\partial^{2} w}{\partial x^{2}}=0 .
\end{gathered}
$$

Third governing equation (17) is a well-known one and represents the equation of motion of the immovable beam with the variable cross-section.

As we can see the boundary conditions and the loading direction does not provide any new aspects to the dynamical analysis and the beam (Fig. 2) can be treated as equivalent to the stationary one. Much more interesting case of the loading is the beam loaded by a transversal time varying force orthogonal to an axis of the beam and simultaneously parallel to the disk's plane. This case (Fig. 3) where the beam with a variable cross-section $A(x)$ and a variable moment of inertia $I(x)$ is considered. 


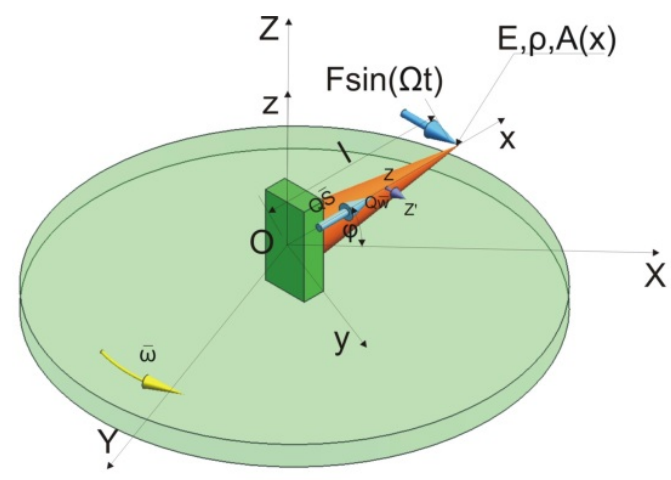

Fig. 3 Model of the analyzed beam with the linearly variable cross-section fixed on the rotational disk and loaded by a transversal force orthogonal to an axis of the beam and parallel to the disk's plane [3]

Analogically to the previous equations of motion it can be derived by means of the classical method. Interesting case of the loading is the beam loaded by a transversal time varying force orthogonal to an axis of the beam and simultaneously parallel to the disk's plane. The obtained equations of motion are coupled ones with the Coriolis elements. After loading the beam in the direction of the $y$ axis of the local reference frame it can be derived the equations of motion of the beam in the matrix form. This system of equations of motion is the fourth order partial differential equations (PDE) and can be expressed as follows:

$$
\begin{aligned}
& \rho A(x)\left[\begin{array}{ccc}
\cos \varphi & -\sin \varphi & 0 \\
\sin \varphi & \cos \varphi & 0 \\
0 & 0 & 1
\end{array}\right]\left[\begin{array}{c}
0 \\
\frac{\partial^{2} w}{\partial t^{2}} \\
0
\end{array}\right]-\rho A(x)\left[\begin{array}{ccc}
\cos \varphi & -\sin \varphi & 0 \\
\sin \varphi & \cos \varphi & 0 \\
0 & 0 & 1
\end{array}\right]\left[\begin{array}{c}
\omega^{2} s \\
\omega^{2} w \\
0
\end{array}\right]+ \\
& -2 \rho A(x)\left[\begin{array}{ccc}
\cos \varphi & -\sin \varphi & 0 \\
\sin \varphi & \cos \varphi & 0 \\
0 & 0 & 1
\end{array}\right]\left[\begin{array}{c}
\omega \frac{\partial w}{\partial t} \\
0 \\
0 \\
0
\end{array}\right]+\rho A(x)\left[\begin{array}{ccc}
\cos \varphi & -\sin \varphi & 0 \\
\sin \varphi & \cos \varphi & 0 \\
0 & 0 & 1
\end{array}\right]\left[\begin{array}{c}
-\dot{\omega} w \\
\dot{\omega} s \\
0
\end{array}\right]= \\
& =-\left[\begin{array}{ccc}
\cos \varphi & -\sin \varphi & 0 \\
\sin \varphi & \cos \varphi & 0 \\
0 & 0 & 1
\end{array}\right]\left[\begin{array}{c}
\frac{\partial^{2}}{\partial x^{2}}\left(\begin{array}{c}
E I(x) \frac{\partial^{2} w}{\partial x^{2}} \\
0
\end{array}\right]
\end{array}\right.
\end{aligned}
$$

in each point of the range $D=\{(x, t), x \mathrm{CE}(0, l), t>0\}$ the Eq. (18) coincides with boundary conditions and initials conditions.

The projection onto the $\mathrm{X}$ and $\mathrm{Y}$ axes, can be obtained, where the projection onto the $\mathrm{X}$ axis of the global reference frame is as follows:

$$
\begin{aligned}
& \rho A(x) \frac{\partial^{2} w_{X}}{\partial t^{2}}+\frac{\partial^{2}}{\partial x^{2}}\left(E I(x) \frac{\partial^{2} w_{X}}{\partial x^{2}}\right)-2 \rho A(x) \omega \frac{\partial w_{Y}}{\partial t}+ \\
& -\rho A(x)\left(\dot{\omega} w_{Y}+\dot{\omega} s \sin \varphi\right)=\rho A(x) \omega^{2}\left(w_{X}+s \cos \varphi\right),
\end{aligned}
$$


and for the $\mathrm{Y}$ axis of the global reference frame, the projection can be written:

$$
\begin{aligned}
& \rho A(x) \frac{\partial^{2} w_{Y}}{\partial t^{2}}+\frac{\partial^{2}}{\partial x^{2}}\left(E I(x) \frac{\partial^{2} w_{Y}}{\partial x^{2}}\right)+2 \rho A(x) \omega \frac{\partial w_{X}}{\partial t}+ \\
& +\rho A(x)\left(\dot{\omega} w_{X}+\dot{\omega} s \cos \varphi\right)=\rho A(x) \omega^{2}\left(w_{Y}+s \sin \varphi\right),
\end{aligned}
$$

where using the real coordinates the individual displacements onto the axes of the global reference frame are:

$$
\begin{aligned}
& w_{X}=-w \sin \varphi \\
& w_{Y}=w \cos \varphi
\end{aligned}
$$

It is obvious that the vibrating beams can be described by different boundary conditions for different loading and fixations types and this case can be treated as the general one.

\section{MATHEMATICAL MODEL OF THE SYSTEM}

The mathematical model of the analyzed beam is provided by the orthogonalization of the equations of motion (19) and (20). The orthogonalization is provided by the Eqs. (19) and (20) which are multiplied using the eigenfunction for the displacements and computing integrals from the equations of motion in the beams limits of integration from the origin of the beam (zero) to the end (a length of the beam). After assuming a constant angular velocity, an angular acceleration equals zero and after introducing Eqs. (21), then the projecting equations of motion Eq. (18) onto the axes of the global reference frame can be written:

$$
\begin{aligned}
& \int_{0}^{l} \rho A(x) \frac{\partial^{2} w_{X}}{\partial t^{2}} X(x) d x+\int_{0}^{l} E I(x) \frac{\partial^{4} w_{X}}{\partial x^{4}} X(x) d x+ \\
& +2 \int_{0}^{l} \frac{\partial E I(x)}{\partial x} \frac{\partial^{3} w_{X}}{\partial x^{3}} X(x) d x+\int_{0}^{l} \frac{\partial^{2} E I(x)}{\partial x^{2}} \frac{\partial^{2} w_{X}}{\partial x^{2}} X(x) d x+ \\
& -2 \rho \int_{0}^{l} A(x) \omega \frac{\partial w_{Y}}{\partial t} X(x) d x-\int_{0}^{l} \rho A(x) \omega^{2} w_{X} X(x) d x=0,
\end{aligned}
$$

and for the second equation, also multiplied by the eigenfunction for displacement and integrated along the beam:

$$
\begin{aligned}
& \int_{0}^{l} \rho A(x) \frac{\partial^{2} w_{Y}}{\partial t^{2}} X(x) d x+\int_{0}^{l} E I(x) \frac{\partial^{4} w_{Y}}{\partial x^{4}} X(x) d x+ \\
& +2 \int_{0}^{l} \frac{\partial E I(x)}{\partial x} \frac{\partial^{3} w_{Y}}{\partial x^{3}} X(x) d x+\int_{0}^{l} \frac{\partial^{2} E I(x)}{\partial x^{2}} \frac{\partial^{2} w_{Y}}{\partial x^{2}} X(x) d x+ \\
& +2 \rho \int_{0}^{l} A(x) \omega \frac{\partial w_{X}}{\partial t} X(x) d x-\int_{0}^{l} \rho A(x) \omega^{2} w_{Y} X(x) d x=0 .
\end{aligned}
$$


The presented mathematical model is universal for different functions describing the variability of the beam's cross-section. The cross-section of the analyzed beam can be now treated as both linearly and nonlinearly variable. If the beam has the nonlinearly variable cross-section (Fig. 4-5) the appropriate function describing this variation ought to be adopted. The different loading terms can be considered as well. For example the beam is loaded by a transversal force orthogonal to an axis of the beam and orthogonal to the disk's plane (Fig. 4) or the beam is loaded by a transversal force orthogonal to an axis of the beam and in parallel to the disk's plane (Fig. 5).

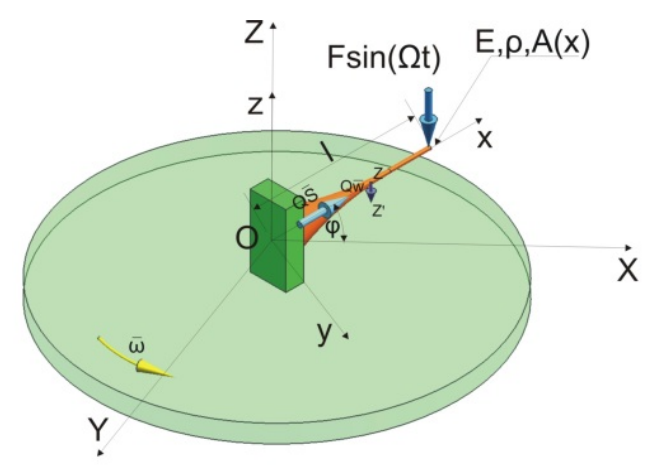

Fig. 4 Model of the analyzed beam with the nonlinearly variable cross-section fixed on the rotational disk and loaded by a transversal force orthogonal to an axis of the beam and orthogonal to the disk's plane

These cases can be considered separately or combined. According to the scientific literature the case presented in figure 4 is well-known in the mathematical sense, likewise the case in figure 1.

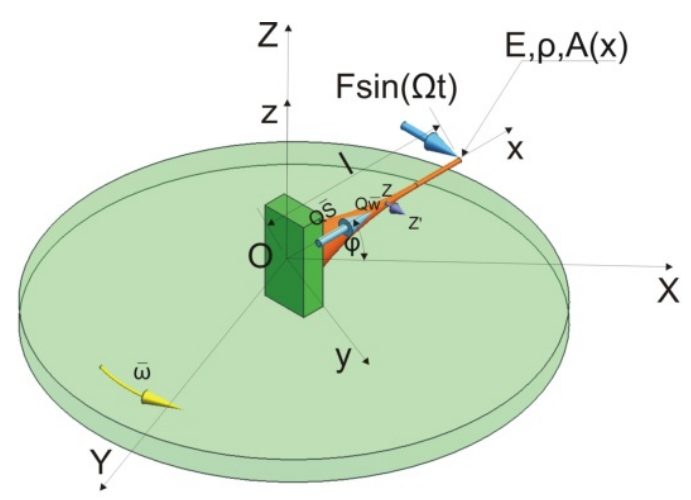

Fig. 5 Model of the analyzed beam with the nonlinearly variable cross-section fixed on the rotational disk and loaded by a transversal force orthogonal to an axis of the beam and in parallel to the plane of the disk

It is assumed that elements of the Eqs. (22) and (23) are the continuously differentiable functions. Using the Eq. (22) and multiple integrating both sides of this equation by parts, the following formula can be obtained: 


$$
\begin{aligned}
& E\left[X(x) I(x) \frac{\partial^{3} w_{X}}{\partial x^{3}}-\frac{\partial X(x) I(x)}{\partial x} \frac{\partial^{2} w_{X}}{\partial x^{2}}+\frac{\partial^{2} X(x) I(x)}{\partial x^{2}} \frac{\partial w_{X}}{\partial x}\right]_{0}^{l}+ \\
& -\left.E \frac{\partial^{3} X(x) I(x)}{\partial x^{3}} w_{X}\right|_{0} ^{l}+E \int_{0}^{l} I(x) X^{I V}(x) w_{X} d x+ \\
& +4 E \int_{0}^{l} I^{\prime}(x) X^{\prime \prime \prime}(x) w_{X} d x+6 E \int_{0}^{l} I^{\prime \prime}(x) X^{\prime \prime}(x) w_{X} d x+ \\
& +4 E \int_{0}^{l} I^{\prime \prime \prime}(x) X^{\prime}(x) w_{X} d x+E \int_{0}^{l} I^{I V}(x) X(x) w_{X} d x+ \\
& +\int_{0}^{l} \rho A(x) \frac{\partial^{2} w_{X}}{\partial t^{2}} X(x) d x+ \\
& +2 E\left[X(x) I^{\prime}(x) \frac{\partial^{2} w_{X}}{\partial x^{2}}-\frac{\partial X(x) I^{\prime}(x)}{\partial x} \frac{\partial w_{X}}{\partial x}+\frac{\partial^{2} X(x) I^{\prime}(x)}{\partial x^{2}} w_{X}\right]_{0}^{l}+ \\
& -2 E \int_{0}^{l} I^{\prime}(x) X^{\prime \prime \prime}(x) w_{X} d x-6 E \int_{0}^{l} I^{\prime \prime}(x) X^{\prime \prime}(x) w_{X} d x+ \\
& -6 E \int_{0}^{l} I^{\prime \prime \prime}(x) X^{\prime}(x) w_{X} d x-2 E \int_{0}^{l} I^{I V}(x) X(x) w_{X} d x+ \\
& +E\left[X(x) I^{\prime \prime}(x) \frac{\partial w_{X}}{\partial x}-\frac{\partial X(x) I^{\prime \prime}(x)}{\partial x} w_{X}\right]_{0}^{l}+E \int_{0}^{l} I^{\prime \prime}(x) X^{\prime \prime}(x) w_{X} d x+ \\
& +2 E \int_{0}^{l} I^{\prime \prime \prime}(x) X^{\prime}(x) w_{X} d x+E \int_{0}^{l} I^{I V}(x) X(x) w_{X} d x+ \\
& -2 \rho \int_{0}^{l} A(x) \omega \frac{\partial w_{Y}}{\partial t} X(x) d x-\int_{0}^{l} \rho A(x) \omega^{2} w_{X} X(x) d x=0,
\end{aligned}
$$

where the variable cross-section $A(x)$ and the moment of inertia $I(x)$ depend on the geometry of the analyzed beam. There is a beam with the linearly variable cross-section Eqs. (7) and (12) assumed for further calculations. The eigenfunction associated with transversal vibrations $X(x)$ of the beam is obtained by applying undamped conditions. Similarly to the first equation, the integration results for the second equation (23) can be applied as follows: 


$$
\begin{aligned}
& E\left[X(x) I(x) \frac{\partial^{3} w_{Y}}{\partial x^{3}}-\frac{\partial X(x) I(x)}{\partial x} \frac{\partial^{2} w_{Y}}{\partial x^{2}}+\frac{\partial^{2} X(x) I(x)}{\partial x^{2}} \frac{\partial w_{Y}}{\partial x}\right]_{0}^{l}+ \\
& -\left.E \frac{\partial^{3} X(x) I(x)}{\partial x^{3}} w_{Y}\right|_{0} ^{l}+E \int_{0}^{l} I(x) X^{I V}(x) w_{Y} d x+ \\
& +4 E \int_{0}^{l} I^{\prime}(x) X^{\prime \prime \prime}(x) w_{Y} d x+6 E \int_{0}^{l} I^{\prime \prime}(x) X^{\prime \prime}(x) w_{Y} d x+ \\
& +4 E \int_{0}^{l} I^{\prime \prime \prime}(x) X^{\prime}(x) w_{Y} d x+E \int_{0}^{l} I^{I V}(x) X(x) w_{Y} d x+ \\
& +\int_{0}^{l} \rho A(x) \frac{\partial^{2} w_{Y}}{\partial t^{2}} X(x) d x+ \\
& +2 E\left[X(x) I^{\prime}(x) \frac{\partial^{2} w_{Y}}{\partial x^{2}}-\frac{\partial X(x) I^{\prime}(x)}{\partial x} \frac{\partial w_{Y}}{\partial x}+\frac{\partial^{2} X(x) I^{\prime}(x)}{\partial x^{2}} w_{Y}\right]_{0}^{l}+ \\
& -2 E \int_{0}^{l} I^{\prime}(x) X^{\prime \prime \prime}(x) w_{Y} d x-6 E \int_{0}^{l} I^{\prime \prime}(x) X^{\prime \prime}(x) w_{Y} d x+ \\
& -6 E \int_{0}^{l} I^{\prime \prime \prime}(x) X^{\prime}(x) w_{Y} d x-2 E \int_{0}^{l} I^{I V}(x) X(x) w_{Y} d x+ \\
& +E\left[X(x) I^{\prime \prime}(x) \frac{\partial w_{Y}}{\partial x}-\frac{\partial X(x) I^{\prime \prime}(x)}{\partial x} w_{Y}\right]_{0}^{l}+E \int_{0}^{l} I^{\prime \prime}(x) X^{\prime \prime}(x) w_{Y} d x+ \\
& +2 E \int_{0}^{l} I^{\prime \prime \prime}(x) X^{\prime}(x) w_{Y} d x+E \int_{0}^{l} I^{I V}(x) X(x) w_{Y} d x+ \\
& +2 \rho \int_{0}^{l} A(x) \omega \frac{\partial w_{X}}{\partial t} X(x) d x-\int_{0}^{l} \rho A(x) \omega^{2} w_{Y} X(x) d x=0 .
\end{aligned}
$$

It is possible to simplify the obtained equations as shown in the Eq. (26). Additionally, after organizing the elements in order, the Eqs. (24) and (25) can be expressed as: 


$$
\begin{aligned}
& E\left[X(x) I(x) \frac{\partial^{3} w_{X}}{\partial x^{3}}-\frac{\partial X(x) I(x)}{\partial x} \frac{\partial^{2} w_{X}}{\partial x^{2}}+\frac{\partial^{2} X(x) I(x)}{\partial x^{2}} \frac{\partial w_{X}}{\partial x}\right]_{0}^{l}+ \\
& -\left.E \frac{\partial^{3} X(x) I(x)}{\partial x^{3}} w_{X}\right|_{0} ^{l}+E \int_{0}^{l} I(x) X^{I V}(x) w_{X} d x+2 E \int_{0}^{l} I^{\prime}(x) X^{\prime \prime \prime}(x) w_{X} d x+ \\
& +\int_{0}^{l} \rho A(x) \frac{\partial^{2} w_{X}}{\partial t^{2}} X(x) d x+E \int_{0}^{l} I^{\prime \prime}(x) X^{\prime \prime}(x) w_{X} d x+ \\
& +2 E\left[X(x) I^{\prime}(x) \frac{\partial^{2} w_{X}}{\partial x^{2}}-\frac{\partial X(x) I^{\prime}(x)}{\partial x} \frac{\partial w_{X}}{\partial x}+\frac{\partial^{2} X(x) I^{\prime}(x)}{\partial x^{2}} w_{X}\right]_{0}^{l}+ \\
& +E\left[X(x) I^{\prime \prime}(x) \frac{\partial w_{X}}{\partial x}-\frac{\partial X(x) I^{\prime \prime}(x)}{\partial x} w_{X}\right]_{0}^{l}+ \\
& -2 \rho \int_{0}^{l} A(x) \omega \frac{\partial w_{Y}}{\partial t} X(x) d x-\int_{0}^{l} \rho A(x) \omega^{2} w_{X} X(x) d x=0
\end{aligned}
$$

and the coupled second equation:

$$
\begin{aligned}
& E\left[X(x) I(x) \frac{\partial^{3} w_{Y}}{\partial x^{3}}-\frac{\partial X(x) I(x)}{\partial x} \frac{\partial^{2} w_{Y}}{\partial x^{2}}+\frac{\partial^{2} X(x) I(x)}{\partial x^{2}} \frac{\partial w_{Y}}{\partial x}\right]_{0}^{l}+ \\
& -\left.E \frac{\partial^{3} X(x) I(x)}{\partial x^{3}} w_{Y}\right|_{0} ^{l}+E \int_{0}^{l} I(x) X^{I V}(x) w_{Y} d x+2 E \int_{0}^{l} I^{\prime}(x) X^{\prime \prime \prime}(x) w_{Y} d x+ \\
& +E \int_{0}^{l} I^{\prime \prime}(x) X^{\prime \prime}(x) w_{Y} d x+\int_{0}^{l} \rho A(x) \frac{\partial^{2} w_{Y}}{\partial t^{2}} X(x) d x+ \\
& +2 E\left[X(x) I^{\prime}(x) \frac{\partial^{2} w_{Y}}{\partial x^{2}}-\frac{\partial X(x) I^{\prime}(x)}{\partial x} \frac{\partial w_{Y}}{\partial x}+\frac{\partial^{2} X(x) I^{\prime}(x)}{\partial x^{2}} w_{Y}\right]_{0}^{l}+ \\
& +E\left[X(x) I^{\prime \prime}(x) \frac{\partial w_{Y}}{\partial x}-\frac{\partial X(x) I^{\prime \prime}(x)}{\partial x} w_{Y}\right]_{0}^{l}+2 \rho \int_{0}^{l} A(x) \omega \frac{\partial w_{X}}{\partial t} X(x) d x+ \\
& -\int_{0}^{l} \rho A(x) \omega^{2} w_{Y} X(x) d x=0 .
\end{aligned}
$$

There are introduced the equivalent notation for the eigenfunctions, cross-sections and the moments of inertia as:

$$
X(x)=X, \quad A(x)=A, \quad I(x)=I .
$$


The Eqs. (26) and (27) after substituting (28) are simplified to:

$$
\begin{aligned}
& E\left[X I \frac{\partial^{3} w_{X}}{\partial x^{3}}-\left(X^{\prime} I+X I^{\prime}\right) \frac{\partial^{2} w_{X}}{\partial x^{2}}+\left(X^{\prime \prime} I+2 X^{\prime} I^{\prime}+X I^{\prime \prime}\right) \frac{\partial w_{X}}{\partial x}\right]_{0}^{l}+ \\
& -\left.E\left(X^{\prime \prime \prime} I+3 X^{\prime \prime} I^{\prime}+3 X^{\prime} I^{\prime \prime}+I^{\prime \prime \prime} X\right) w_{X}\right|_{0} ^{l}+E \int_{0}^{l} I X^{I V} w_{Y} d x+ \\
& +2 E \int_{0}^{l} I^{\prime} X^{\prime \prime \prime} w_{X} d x+E \int_{0}^{l} I^{\prime \prime} X^{\prime \prime} w_{X} d x+\int_{0}^{l} \rho A \frac{\partial^{2} w_{X}}{\partial t^{2}} X d x+ \\
& +E\left[2 X I^{\prime} \frac{\partial^{2} w_{X}}{\partial x^{2}}-2\left(X^{\prime} I^{\prime}+X I^{\prime \prime}\right) \frac{\partial w_{X}}{\partial x}+2\left(X^{\prime \prime} I^{\prime}+2 X^{\prime} I^{\prime \prime}+I^{\prime \prime \prime} X\right) w_{X}\right]_{0}^{l}+ \\
& +E\left[X I^{\prime \prime} \frac{\partial w_{X}}{\partial x}-\left(X^{\prime} I^{\prime \prime}+X I^{\prime \prime \prime}\right) w_{X}\right]_{0}^{l}-2 \rho \int_{0}^{l} A \omega \frac{\partial w_{Y}}{\partial t} X d x-\int_{0}^{l} \rho A \omega^{2} w_{X} X d x=0
\end{aligned}
$$

and analogically to the second equation (27) for the $\mathrm{Y}$ axis of the global reference frame can be obtained as:

$$
\begin{aligned}
& E\left[X I \frac{\partial^{3} w_{Y}}{\partial x^{3}}-\left(X^{\prime} I+X I^{\prime}\right) \frac{\partial^{2} w_{Y}}{\partial x^{2}}+\left(X^{\prime \prime} I+2 X^{\prime} I^{\prime}+X I^{\prime \prime}\right) \frac{\partial w_{Y}}{\partial x}\right]_{0}^{l}+ \\
& -\left.E\left(X^{\prime \prime \prime} I+3 X^{\prime \prime} I^{\prime}+3 X^{\prime} I^{\prime \prime}+I^{\prime \prime \prime} X\right) w_{Y}\right|_{0} ^{l}+E \int_{0}^{l} I X^{I V} w_{Y} d x+ \\
& +2 E \int_{0}^{l} I^{\prime} X^{\prime \prime \prime} w_{Y} d x+E \int_{0}^{l} I^{\prime \prime} X^{\prime \prime} w_{Y} d x+\int_{0}^{l} \rho A \frac{\partial^{2} w_{Y}}{\partial t^{2}} X d x+ \\
& +E\left[2 X I^{\prime} \frac{\partial^{2} w_{Y}}{\partial x^{2}}-2\left(X^{\prime} I^{\prime}+X I^{\prime \prime}\right) \frac{\partial w_{Y}}{\partial x}+2\left(X^{\prime \prime} I^{\prime}+2 X^{\prime} I^{\prime \prime}+I^{\prime \prime \prime} X\right) w_{Y}\right]_{0}^{l}+ \\
& +E\left[X I^{\prime \prime} \frac{\partial w_{Y}}{\partial x}-\left(X^{\prime} I^{\prime \prime}+X I^{\prime \prime \prime}\right) w_{Y}^{l}+2 \rho \int_{0}^{l} A \omega \frac{\partial w_{X}}{\partial t} X d x-\int_{0}^{l} \rho A \omega^{2} w_{Y} X d x=0 .\right.
\end{aligned}
$$

The Eqs. (29) and (30) can be simplified as well and the following two equations yield: 


$$
\begin{aligned}
& E\left[X I \frac{\partial^{3} w_{X}}{\partial x^{3}}-\left(X^{\prime} I-X I^{\prime}\right) \frac{\partial^{2} w_{X}}{\partial x^{2}}+X^{\prime \prime} I \frac{\partial w_{X}}{\partial x}\right]_{0}^{l}+ \\
& -\left.E\left(X^{\prime \prime \prime} I+X^{\prime \prime} I^{\prime}\right) w_{X}\right|_{0} ^{l}+E \int_{0}^{l} I X^{I V} w_{X} d x+ \\
& +2 E \int_{0}^{l} I^{\prime} X^{\prime \prime \prime} w_{X} d x+E \int_{0}^{l} I^{\prime \prime} X^{\prime \prime} w_{X} d x+\int_{0}^{l} \rho A \frac{\partial^{2} w_{X}}{\partial t^{2}} X d x+ \\
& -2 \rho \int_{0}^{l} A \omega \frac{\partial w_{Y}}{\partial t} X d x-\int_{0}^{l} \rho A \omega^{2} w_{X} X d x=0,
\end{aligned}
$$

the second equation (30) additionally after simplifying the integrals:

$$
\begin{aligned}
& E\left[X I \frac{\partial^{3} w_{Y}}{\partial x^{3}}-\left(X^{\prime} I-X I^{\prime}\right) \frac{\partial^{2} w_{Y}}{\partial x^{2}}+X^{\prime \prime} I \frac{\partial w_{Y}}{\partial x}\right]_{0}^{l}+ \\
& -\left.E\left(X^{\prime \prime \prime} I+X^{\prime \prime} I^{\prime}\right) w_{Y}\right|_{0} ^{l}+E \int_{0}^{l} \frac{\partial^{2} I X^{\prime \prime}}{\partial x^{2}} w_{Y} d x+\int_{0}^{l} \rho A \frac{\partial^{2} w_{Y}}{\partial t^{2}} X d x+ \\
& +2 \rho \int_{0}^{l} A \omega \frac{\partial w_{X}}{\partial t} X d x-\int_{0}^{l} \rho A \omega^{2} w_{Y} X d x=0 .
\end{aligned}
$$

By introducing the acting external force, it can be noticed:

$$
\begin{aligned}
& E I \frac{\partial^{3} w_{X}(l)}{\partial x^{3}}-E I^{\prime} \frac{\partial^{2} w_{X}(l)}{\partial x^{2}}=0 \\
& E I \frac{\partial^{3} w_{Y}(l)}{\partial x^{3}}-E I^{\prime} \frac{\partial^{2} w_{Y}(l)}{\partial x^{2}}=-2 \int_{0}^{l} F_{0} \delta(x-l) \cos (\Omega t) d x .
\end{aligned}
$$

Based on the boundary conditions (11) it can be written:

$$
\begin{aligned}
& \frac{\partial^{2} w_{X}(l)}{\partial x^{2}}=\frac{\partial^{2} w_{Y}(l)}{\partial x^{2}}=0, \\
& X I \frac{\partial^{3} w_{X}(0)}{\partial x^{3}}-\frac{\partial X I}{\partial x} \frac{\partial^{2} w_{X}(0)}{\partial x^{2}}=0, \\
& X I \frac{\partial^{3} w_{Y}(0)}{\partial x^{3}}-\frac{\partial X I}{\partial x} \frac{\partial^{2} w_{Y}(0)}{\partial x^{2}}=0
\end{aligned}
$$


and

$$
\begin{aligned}
& E\left[X^{\prime \prime} I \frac{\partial w_{X}}{\partial x}-\frac{\partial X^{\prime \prime} I}{\partial x} w_{X}\right]_{0}^{l}=0, \\
& E\left[X^{\prime \prime} I \frac{\partial w_{Y}}{\partial x}-\frac{\partial X^{\prime \prime} I}{\partial x} w_{Y}\right]_{0}^{l}=0 .
\end{aligned}
$$

By introducing (34-35) the first equation yields:

$$
\begin{aligned}
& E \int_{0}^{l} I X^{I V} w_{X} d x+2 E \int_{0}^{l} I^{\prime} X^{\prime \prime \prime} w_{X} d x+E \int_{0}^{l} I^{\prime \prime} X^{\prime \prime} w_{X} d x+\int_{0}^{l} \rho A \frac{\partial^{2} w_{X}}{\partial t^{2}} X d x+ \\
& -2 \rho \int_{0}^{l} A \omega \frac{\partial w_{Y}}{\partial t} X d x-\int_{0}^{l} \rho A \omega^{2} w_{X} X d x=0 .
\end{aligned}
$$

Where the searched solutions are defined as displacements in the global reference frame and the ones are separable in time and space domains. The system is symmetric and the motion of the analyzed cross-section is given by the multiplication of the projected harmonic time dependent eigenfunctions with the frequency $\Omega$ and the eigenfunctions for displacements with amplitudes XY. Both the variables coincide with the responses of two decoupled harmonic oscillators. In the present case the searched solutions are:

$$
\begin{aligned}
& w_{X}=\sum_{n=1}^{\infty} a_{X} X(x) \sin (\Omega t), \\
& w_{Y}=\sum_{n=1}^{\infty} a_{Y} X(x) \cos (\Omega t) .
\end{aligned}
$$

The norm for Eqs. (36) is assumed as:

$$
\gamma_{n}^{2}=\int_{0}^{l} A X^{2} d x
$$

for the beam with the linearly variable cross-section (Fig. 3) the norm is calculated as integral.

Both $I_{1}$ and $I_{2}$ coefficients are connected with the moments of inertia and the eigenfunctions for displacements. The I and c (velocity) coefficients are as follows: 


$$
\begin{aligned}
& I_{1}=\frac{1}{\gamma_{n}^{2}} \int_{0}^{l} I X^{2} d x, \\
& I_{2}=\frac{1}{\gamma_{n}^{2}} \int_{0}^{l} I^{\prime \prime} X^{2} d x, \\
& c=\sqrt{\frac{E}{\rho}} .
\end{aligned}
$$

It can be also noticed that:

$$
\int_{0}^{l} X^{\prime \prime \prime} I^{\prime} d x=0
$$

The $a$ coefficients (amplitudes) can be obtained as:

$$
\begin{aligned}
& a_{X}=\frac{-2 \omega \Omega F_{0} X(l)}{\rho \gamma_{n}^{2}\left[c^{4} k^{4}\left(I_{2}-I_{1} k^{2}\right)^{2}+\left(\omega^{2}-\Omega^{2}\right)^{2}+2 c^{2} k^{2}\left(I_{2}-I_{1} k^{2}\right)\left(\omega^{2}+\Omega^{2}\right)\right]} \\
& a_{Y}=\frac{F_{0} X(l)}{2 \rho \gamma_{n}^{2}}\left\{\frac{-1}{I_{2} k^{2}-c^{2} I_{1} k^{4}+\left(\omega^{2}-\Omega^{2}\right)^{2}}-\frac{1}{I_{2} k^{2}-c^{2} I_{1} k^{4}+\left(\omega^{2}+\Omega^{2}\right)^{2}}\right\}
\end{aligned}
$$

Knowing these amplitudes the total displacements can be calculated and the solution in the global reference frame can be obtained.

\section{DYNAMICAL FLEXIBILITIES}

According to the author the dynamical flexibility is understood as the amplitude of generalized displacement in the direction of " $\mathrm{i}$ " generalized coordinate changed by generalized harmonic force with amplitude equals 1 in the direction of "k" generalized coordinate. This definition can be written in the mathematical form as:

$$
{ }_{1} s_{i}=Y_{i k}{ }_{2} s_{k} .
$$

Finally the dynamical flexibility of the rotating beam with the variable cross-section is presented as:

$$
Y=\sum_{n=1}^{\infty} \frac{1}{2 \rho \gamma_{n}^{2}}\left\{\frac{-X(x) X(l)}{I_{2} k^{2}-c^{2} I_{1} k^{4}+\left(\omega^{2}-\Omega^{2}\right)^{2}}-\frac{X(x) X(l)}{I_{2} k^{2}-c^{2} I_{1} k^{4}+\left(\omega^{2}+\Omega^{2}\right)^{2}}\right\}
$$


For comparison, the dynamical flexibility of the rotating beam with a constant cross-section is as follows:

$$
Y_{g}=\sum_{n=1}^{\infty} \frac{X(x) X(l)\left(c_{g}^{2} k^{4}-\omega^{2}-\Omega^{2}\right)}{\rho A_{\text {const }} \gamma_{g n}^{2}\left[c_{g}^{4} k^{8}+\left(\omega^{2}-\Omega^{2}\right)^{2}-2 c_{g}^{2} k^{4}\left(\omega^{2}+\Omega^{2}\right)\right]}
$$

On the other side the dynamical flexibility of the immovable beam is presented as:

$$
Y_{g}=\sum_{n=1}^{\infty} \frac{X(x) X(l)}{\rho A_{\text {const }} \gamma_{g n}^{2}\left(c_{g}^{2} k^{4}-\Omega^{2}\right)} .
$$

where for the Eqs. (44) and (45):

$$
c_{g}=\sqrt{\frac{E I}{\rho A_{\text {const }}}}
$$

and the norm is:

$$
\gamma_{g n}^{2}=\int_{0}^{l} X^{2} d x
$$

Both the derived dynamical flexibilities, the Eq. (44) and Eq. (46), are presented in figures (Fig. 6-7). For numerical examples the beam of $1 \mathrm{~m}$ length is assumed. The beam is the clamped-free one and fixed on the rotational disk. Distinctively from the beam with a constant cross-section, the beam with the variable cross-section has different inconstant bifurcations for the individual modes of vibrations (Fig. 7).

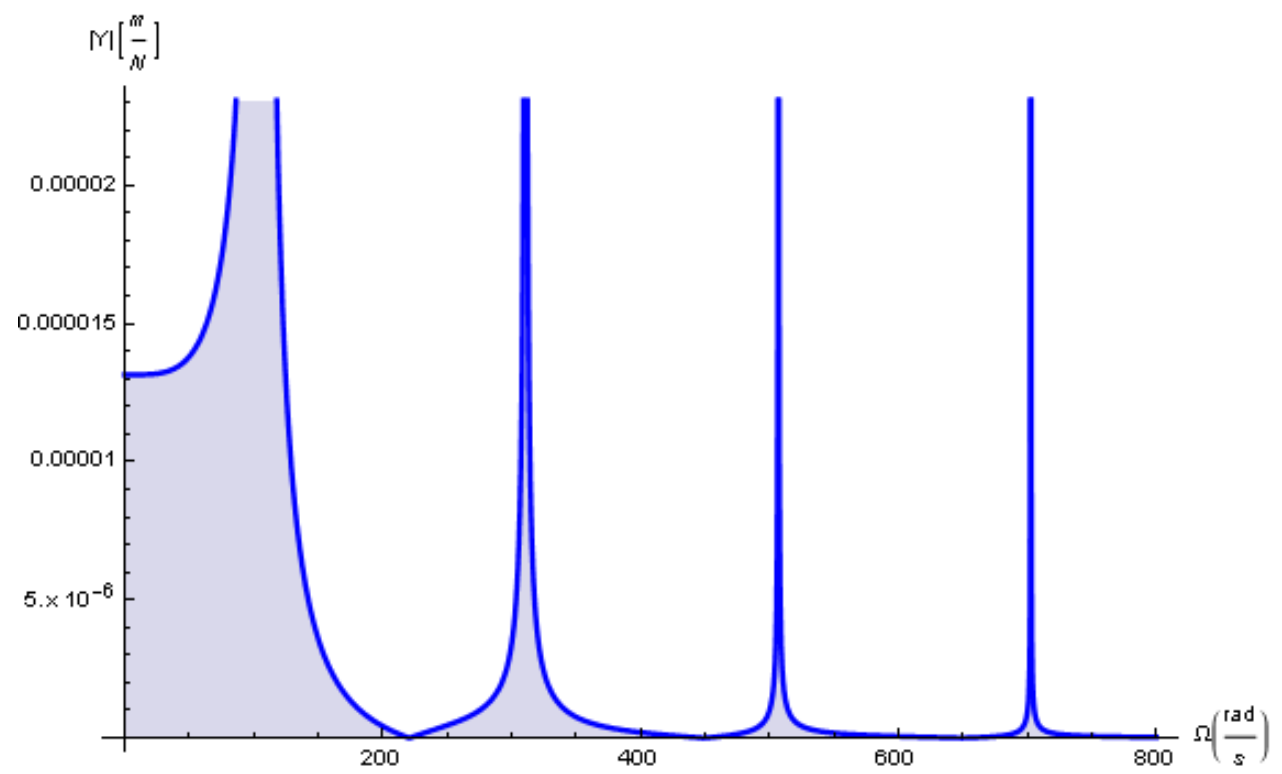

Fig. 6 Sample dynamical flexibility of the analyzed immovable beam with the variable cross-section 


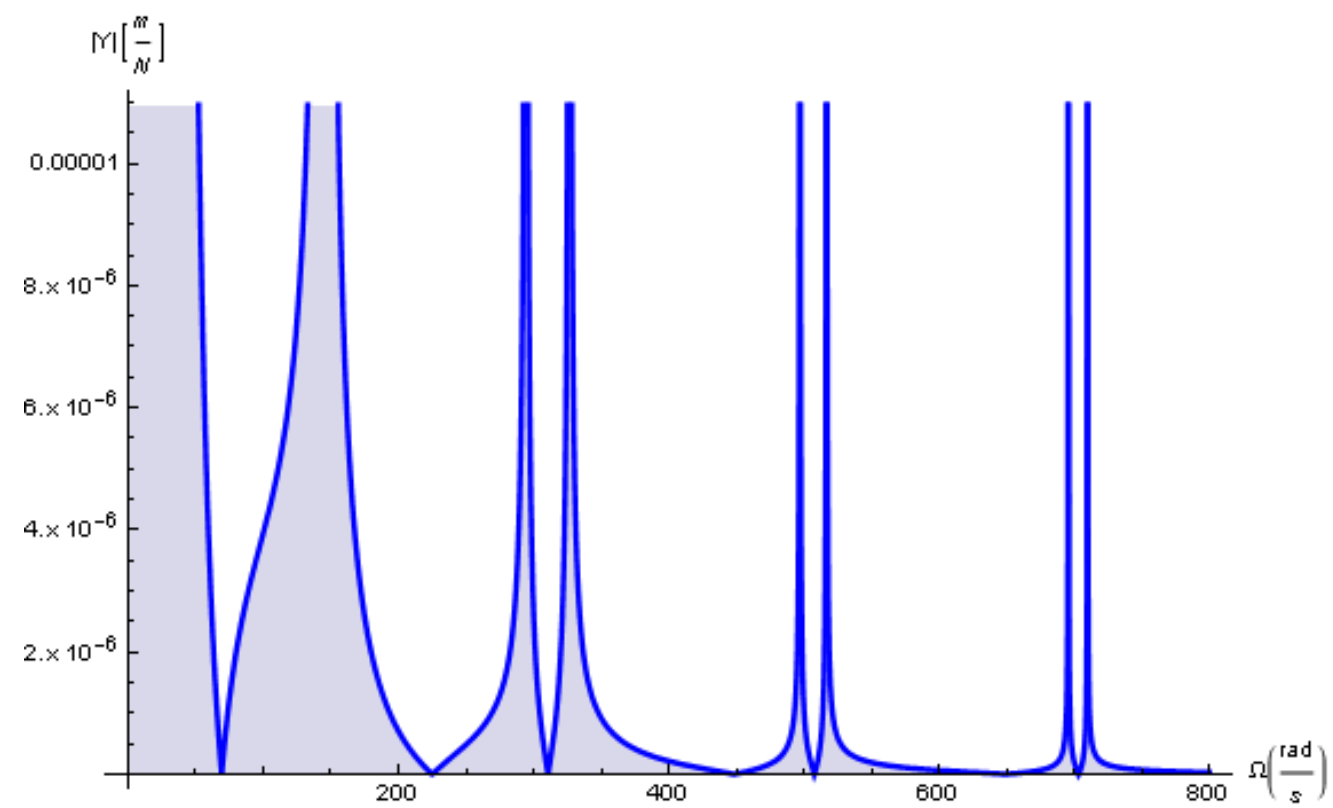

Fig. 7 Dynamical flexibility of the rotating beam with the variable cross-section. The disk is being rotated with the angular velocity equals 100 radians per second

Derivations of suitable dynamical flexibilities are applied to the systems in the transportable motion both vibrating longitudinally and transversally. The solutions can be presented on charts of amplitude (dynamical flexibility) in function of frequency. There are many numerical applications that can be used for this purpose, e.g. GENTA'S DynRot or author's program the Modyfit [18].

\section{Conclusions}

The paper concerns a very crucial problem of beams vibrating in transportation with the variable geometric parameters such as: tapered or coned beams, blades with various shape functions, and others. The analyzed systems could be applied in many technical implementations, obviously after proper adjustment of mathematical models and considering the necessary parameters and conditions. The most popular applications could be connected with pumps and rotors; especially the high speed ones, blades of helicopters and arms of robots. The obtained solutions were also presented in the form of equations of motion. The dynamical analysis was provided using the dynamical flexibility method and the Galerkin's method. The dynamical flexibility method applies solutions presentation as the dynamical characteristics of amplitude in the function of frequency. The solutions were determined as the compact mathematical formulae. The dynamical characteristics were presented on charts. The transportation effect and shape functions describing the cross-section variation were taken into consideration. The centrifugal forces and the Coriolis forces were taken into consideration in the obtained mathematical model. The natural frequencies and the minimal amplitudes can be easily read from the dynamical flexibility characteristics. In these characteristics the beam with the constant cross-section and the beam with the variable cross-section are put together. The juxtaposition of the rotating beam and the immovable one is presented. The results presented are very significant and enable controlling of such a type rotating systems. In future works more numerical exam- 
ples for beamlike systems with the variable cross-section will be presented and the rotating beam with damping forces considered will be analyzed. The rotating beam with some imperfections and defects or cracks can be the potential material for the further analysis and research.

Acknowledgments: the paper was presented during 11th Conference on Dynamical Systems - Theory and Applications.

\section{References}

Al-Ansary M. D.: Flexural vibrations of rotating beams considering rotary inertia. Computers and Structures, 69, 1998, 321-328.

Auciello N. M.: Comment on. A note on vibrating tapered beams. Journal of Sound and Vibration, 1995, 187-724.

Bokhonsky A. I., Zolkiewski S.: Modelling and Analysis of Elastic Systems in Motion. Monograph, Gliwice, Silesian University of Technology Press, 2011, p. 171.

Chang T.-P., Chang H.-C.: Vibration and buckling analysis of rectangular plates with nonlinear elastic end restraints against rotation. International Journal of Solids and Structures, 34, 18, June 1997, 2291-2301.

Cheung Y. K., Zhou D.: The free vibrations of tapered rectangular plates using a new set of beam functions with the Rayleigh-Ritz method. Journal of Sound and Vibration, 223, 5, 1999, 703-722.

Dyniewicz B., Bajer C. I.: New feature of the solution of a Timoshenko beam carrying the moving mass particle. Archives of Mechanics, 62, 5, 2010, 327-341.

Genta G.: Dynamics of Rotating Systems. New York, Springer, 2005.

Grossi RO, Bhat RB. A note on vibrating tapered beams. Journal of Sound and Vibration 1991, 147-174.

Gunda J. B., Gupta R. K., Gangul R.: Hybrid stiff-string-polynomial basis functions for vibration analysis of high speed rotating beams. Computers and Structures, 87, 2009, 254-265.

Kumar R., Kansal T.: Effect of rotation on Rayleigh waves in an isotropic generalized thermoelastic diffusive halfspace. Archives of Mechanics, 60, 5, 2008, 421-443.

Ozturk H.: In-plane free vibration of a pre-stressed curved beam obtained from a large deflected cantilever beam. Finite Elements in Analysis and Design, 47, 2011, 229-236.

Rahman M. A., Kowser M. A., Hossain S. M. M.: Large deflection of the cantilever steel beams of uniform strength experiment and nonlinear analysis. International Journal of Theoretical and Applied Mechanics, 1, 2006, 21-36.

Solecki R., Szymkiewicz J.: The rod and surface systems. Warszawa, Arkady Budownictwo, Sztuka, Architektura, 1964 (in Polish).

Simsek M.: Non-linear vibration analysis of a functionally graded Timoshenko beam under action of a moving harmonic load. Composite Structures, 92, 2010, 2532-2546.

Szefer G.: Dynamics of elastic bodies undergoing large motions and unilateral contact. Journal of Technical Physics. XLI, 4, 2000.

Szefer G.: Dynamics of elastic bodies in terms of plane frictional motion. Journal of Theoretical and Applied Mechanics, 2, 39, 2001.

Zhou D.: Vibrations of point-supported rectangular plates with variable thickness using a set of static tapered beam functions. International Journal of Mechanical Sciences, 44, 2002, 149-164.

Zolkiewski S.: Numerical Application for Dynamical Analysis of Rod and Beam Systems in Transportation. Solid State Phenomena, 164, 2010, 343-348.

Zolkiewski S.: Dynamic flexibility of the supported-clamped beam in transportation. Journal of Vibroengineering. 13, 4, December 2011, 810-816.

Zolkiewski S.: Dynamical Flexibility of Complex Damped Systems Vibrating Transversally in Transportation. Solid State Phenomena, 164, 2010 339-342.

Zolkiewski S.: Damped Vibrations Problem Of Beams Fixed On The Rotational Disk. International Journal of Bifurcation and Chaos, 21, 10, October 2011, 3033-3041.

Zone-Ching L., Don-Tsun L.: Dynamic deflection analysis of a planar robot Computers and Structures, 53, 4, 17 November

1994 ,

947-960. 